\title{
Integrative approach for teaching histology and histopathology of the gastrointestinal tract using an IBD
}

\section{case}

\author{
Inaya Hajj Hussein ${ }^{1}$, Mazen Toushan², Mitual Amin ${ }^{1,2}$ and Ann Marie Blenc ${ }^{1,2}$ \\ ${ }^{1}$ Department of Biomedical Sciences, Oakland University William Beaumont School of Medicine, Rochester, MI, USA \\ ${ }^{2}$ Department of Clinical Pathology, William Beaumont Hospital, Royal Oak, MI, USA
}

\begin{abstract}
Integration has progressed beyond the basic concept of horizontal and vertical integration. We now emphasize student-directed learning, active learning, development of interpersonal skills, problem solving, and self-reflection. Inflammatory Bowel disease (IBD) serves as an excellent model for how we accomplish integration of histology with other disciplines, resulting in one course that is organ-system based, thus increasing the connection to clinical medicine. It has two major constituents: ulcerative colitis and Crohn's disease. It can involve any segment of the GI tract, as well as other systems that share clinical and pathological characteristics.

We use clinical scenarios of IBD cases in order to make histology more clinically relevant. Students address comprehensively the anatomy, histology, histopathology, and pathophysiology in a manner that provides a thorough understanding of the disease. Then they are able to establish a final diagnosis. This approach is facilitated by making available to the students a wide variety of resources.

This student-directed strategy requires a major shift in the way educators think about medical school teaching. It is a successful integration approach whereby students themselves take initiative and responsibility for determining what is worthwhile to learn. In addition to horizontal integration and vertical integration, it offers several intangible benefits. Chief among those benefits is building a community of students with high level of skills and professionalism.
\end{abstract}

\section{Introduction}

For the past 5 years, the new medicine program at Oakland University William Beaumont (OUWB) School of Medicine has been very dynamic and under a continuous clinical review. It employs mostly scenario-based learning with vertically integrated classes for year 1 and year 2 students. Simultaneously, horizontally integrated teaching is presented in this way: a core anatomy course as a discipline specific course, complemented with, refreshing anatomy visits to the various organ systems at the proper time. Reform efforts to the curriculum are always encouraged. However, since beginning, the curriculum and its implementation has increased emphasis on independent learning, professionalism, development of interpersonal skills, and problem solving. These reform efforts are in accordance with the objectives of the General Medical Council which stresses on reduction in contact hours especially with the continuously increasing number of facts[1].

With respect to histology, the trend is in the direction of integrating histology, along with the various other disciplines into one course that is organ-system based. This increases the connection to clinical medicine by using clinical scenarios and making histology more clinically relevant.

The current medical curriculum at OUWB has progressed beyond the basic concept of horizontal and vertical integration. It requires a major shift in the way educators think about medical school teaching and emphasizes student directed learning, student-directed strategies, self-reflection and team based learning. This emphasis goes back to the well-studied and practiced adult learning theories which constitute self-directed learning as one of its fundamental concepts; whereby, self-directed learning promotes autonomy and independence with the teacher acting as a facilitator [2].

It is well known that the teaching of anatomical sciences (gross anatomy, histology and embryology) is undergoing evolution, in particular with the introduction of new information technology resources, such as the virtual man and women, virtual microscopy, 3-D ultrasound and other radiological techniques. The current educational trend for anatomical sciences is towards delivery of reduced information, in a core curriculum that allows integration and alignment of teaching with other basic science courses and clinical scenarios. These integrated approaches (e.g. histology/histopathology) to learning basic sciences provide students with the benefit of developing the necessary skills that are required during subsequent clinical training, stressing on functional and clinical relevance. This horizontal and vertical integration being used by the new curriculum better promotes the acquisition of basic science knowledge through clinical scenarios thus leading to long-term retention and analytic clinical reasoning [3].

The selection of clinical case scenarios is very important, especially

Correspondence to: Inaya Hajj Hussein, Department of Biomedical Sciences, Oakland University William Beaumont School of Medicine, Rochester, MI, USA, Tel: +12488217230; E-mail: hajjhuss@oakland.edu

Key words: integrated learning, histology, histopathology, inflammatory bowel disease

Received: January 12, 2017; Accepted: March 03, 2017; Published: March 06 2017 
when it integrates multiple disciplines together, which allows the students to see the big picture. Inflammatory Bowel Disease (IBD) is a good example of a clinical situation where multiple and comprehensive integrations of various basic sciences can take place. Such integration increases interdisciplinary connections between basic and clinical sciences and enhances clinical relevance.

Clinical cases like IBD, be it Crohn disease (CD) or Ulcerative Colitis (UC), constitute an excellent prototype of clinical scenarios to study the anatomy, histology, physiology, microbiology, immunology, pathology and pathophysiology of the GI tract.

The study of an IBD case can cover all the aspects of anatomy from gross down to the cellular and molecular levels, and can cover both healthy and diseased GI tracts. This approach is facilitated by the availability of online resources covering the various aspects of IBD. Such resources include virtual microscopy, radiographic anatomy, plastinated specimens, pathology specimens, etc. The role of the professor is that of a facilitator, rather than the source of information. He or she is a coordinator and moderator, and the presence of more than one discipline representative in a particular session is highly encouraged.

This article aims to demonstrate the use of an inflammatory bowel disease scenario, to successfully integrate the histology of the GI tract with its histopathology in a single case. It is preferred to be attended by a histologist and a histopathologist. In addition, the objectives of this prototype of integration 'should be aligned with the institutional set of curricular requirements.

\section{Methods}

The implementation of the session includes a series of steps, which starts by selecting the clinical scenario of the inflammatory bowel disease case, involving the various segments of the gastrointestinal tract (GI).

Here is an example of such a scenario

\section{Step 1.}

\section{Case: A man with recurrent diarrhea}

Chris is a 17 -year-old male who presented to his primary care physician with a 6-month history of recurrent mucous diarrhea, abdominal cramps, fever, and weight loss; he also reports occasional blood in his stools.

Medical history: His medical history revealed nothing remarkable and the patient uses no medications.

Physical exam: The patient was noted to have an oral ulcer and a perianal fistula.

\section{Medications: none}

\section{Laboratory investigation:}

1. Stool studies showed negative for salmonella/ shigella \& ova/ parasite \& clostridium difficile toxin

2. Blood tests exhibited mild iron deficiency anemia with increased serum inflammatory markers (ESR \& CRP)

3. Colonoscopy \& biopsy: Images provided

Step 2.
The second step aims at defining and determining the student learning objective of the particular session (e.g. histology and histopathology of the GI). These objectives are in line with the required course objectives. The objectives are as follows:

By the end of this case, the students will be able to:

1. Describe and differentiate between the normal histology of various components of the Gastrointestinal System (GI).

2. Compare the normal histology to its pathological counterpart.

3. Recognize the typical presentations of IBD and its clinical symptoms and signs.

\section{Step 3.}

The third step focuses on identifying the necessary resources (material and human) needed in line with the educational strategies to be used in order to reach the set objectives.

\section{Virtual microscopy slides}

In histology-histopathology information technology is often instrumental in making new resources available and accessible. Actually, most curriculum resource requirements are technologybased and include provision and availability of computer facilities and networks, deployment systems, and technical support. Technology is well employed in this case; it helps in compensating for any material resources deficit. The digital image bank substitutes for microscopy slides. The digital images are delivered through a network system for onsite and offsite online access with a computer digital device. Among the resources available are normal and pathological fixed, prosected or plastinated specimens. They help correlate the microscopic alterations with the gross appearance of the various specimens representing the respective segments of the GI tract.

Furthermore, multiple audiovisual and radiographic images of the GI, both normal and pathological, are made available on the Moodle system. In addition there are colonoscopy views (movies and photos) that can be reviewed, thus enhancing the learning process of the basic and clinical aspects of the IBD in normal and diseased tissues.

\section{Step 4.}

The fourth step is to prepare and propose reading assignments to cover the requirements and propose links with further online resources for a comprehensive preparation before the session. In preparing for the case, students are asked to go over comprehensive assignments, including, previously mentioned resources, some readings assignments and some optional links to online resources directly relevant to the GI histology/histopathology. This assignment can be enhanced by preparing pre-session questions that stimulate or facilitate learning.

\section{Step 5.}

The fifth step deals with implementation of the session. It is accomplished by dividing students into groups in the presence of a histologist and a pathologist. Those instructors will facilitate learning, answer questions and build an interactive session. Students analyze and discuss the clinical scenarios, stressing histological features and corresponding pathological alterations. Each group reaches a possible diagnosis based on those discussions and a set of previously prepared questions. The presence of the histologist and of the pathologist enrich the discussions, since they facilitate for learning. They lead, and even initiate, interactive teaching by asking relevant questions that are in line with the objectives, and they moderate the discussions. Examples 
of such questions could include:

1. What's the highest on your list of differential diagnosis and why?
A. Crohn's disease
B. Gastroenteritis
C. Irritable bowel syndrome
D. Ulcerative colitis

\section{Answer:}

A. Patient is presenting with the classic presentation of IBD: recurrent mucous diarrhea, melena, abdominal cramps, fever, weight loss, oral ulcer, and anemia. The age of the patient (vs. Ulcerative Colitis where patients tend to be $>40$ years of age) and the presence of a perianal fistula are more suggestive of CD. However, UC is also high on the differential diagnosis.

B. Gastroenteritis can be ruled out given the negative stool studies.

C. Irritable bowel syndrome is a diagnosis of exclusion and generally does not present with bloody diarrhea.

D. Most likely the correct diagnosis

2. What other tests/procedures can confirm the diagnosis?

Answer: Colonoscopy with biopsy to rule in/out IBD (CD/ UC)

3. How did the colonoscopy findings help you reach a diagnosis? (Compare with normal counterpart).

Answer: Skipped lesions, patchy erythema, linear ulcers, apthous ulcers, and cobblestone appearance of the mucosa are diagnostic for CD (Figure 1a-1c).

4. Did the biopsy findings help you in confirming the diagnosis? (Compare that to your normal histology and use slidepath images and/or ppt slides to demonstrate the normal structures) (Figure 2a-2c).

5. According to the biopsy, identify which intestinal layers are usually affected in this disease. Compare with your normal histology. (Use slidepath images and/or ppt slides to demonstrate the normal structures.)

Answer: CD presents as a "trasmural" inflammation, i.e. it infects all the layers of the bowel of the intestine (mucosa, submucosa, muscularis externa and serosa).

6. In your opinion, how can imaging studies (e.g.: $x$-ray, CT, MR enterography, and barium studies) help in identifying pathological

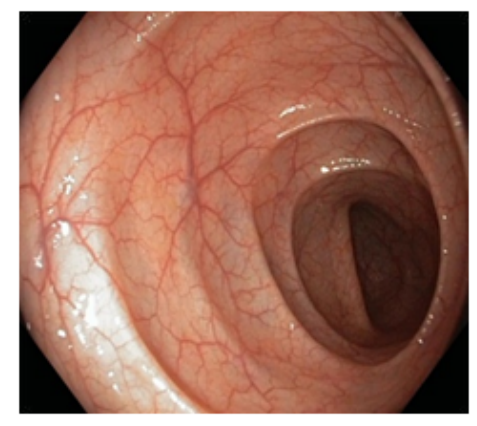

Figure 1a. Normal colonic mucosa.

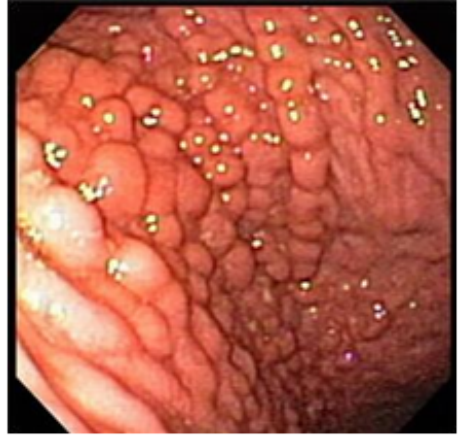

Figure 1b. Cobblestone appearance.

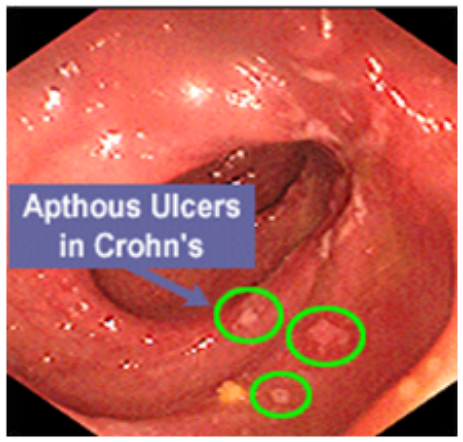

Figure 1c. Apthous ulcers in Crohn's.

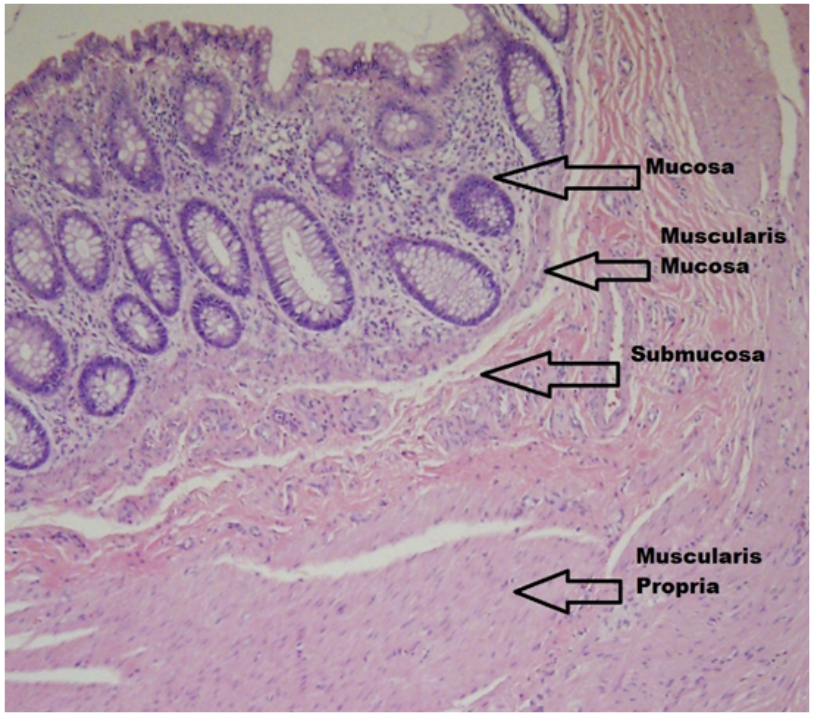

Figure 2a. Normal colonic histology slide.

findings from an anatomical view? Describe at least 3 radiologic findings that are characteristic of this disease (use radiographic images to demonstrate the findings). (Figure $3 a-3 c$ ).

7. After reviewing the results of the tests performed, what is the treatment of this condition?

Answer: Treatment of Crohn's disease aims at reducing inflammation, reliveing symptoms of pain and diarrhea, and eliminating nutritional deficiencies. Treatment options include drugs, nutritional supplements, surgery, or combination. Medications include sulfasalazine, mesalamine, corticosteroids, immunosuppressants, and biological drugs.

8. What are the risk factors for this disease? 


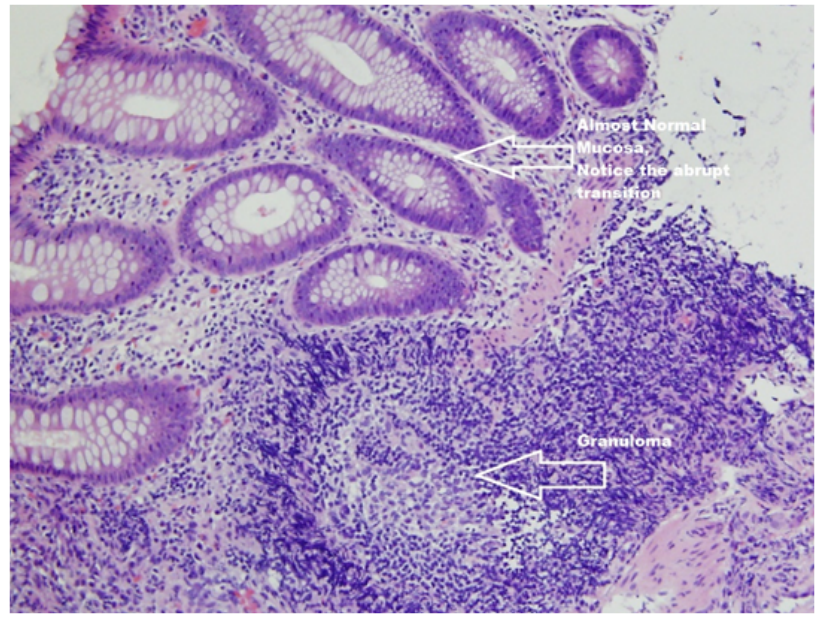

Figure 2b. Pathologic state in Crohn's showing a granuloma.

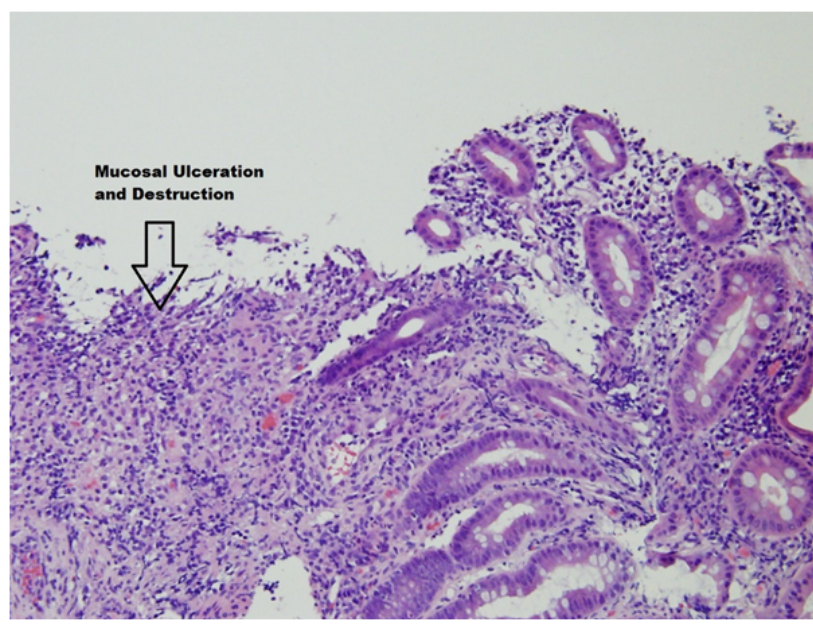

Figure 2c. Pathologic state in Crohn's showing mucosal ulceration and destruction.

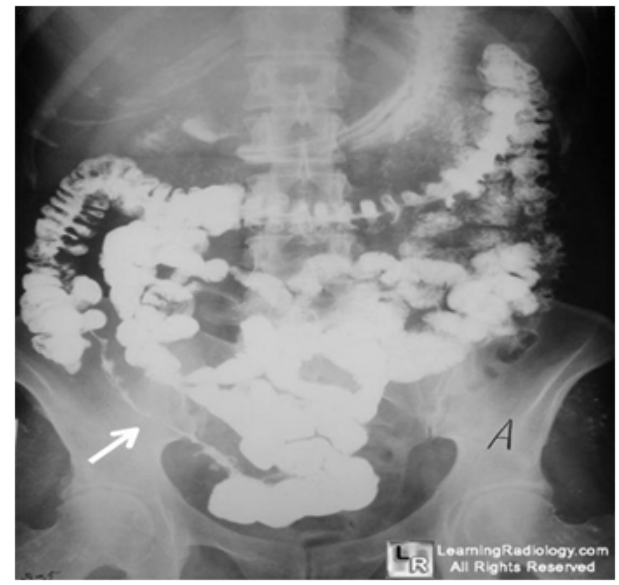

Figure 3a. White arrow showing string sign of the terminal ileum due to narrowing.

Answer: Risk factors for Crohn's disease include: ethnicity, family history, environment and diet high in fat and refined food, and smoking.

9. Explain the pathophysiology behind the watery diarrhea that

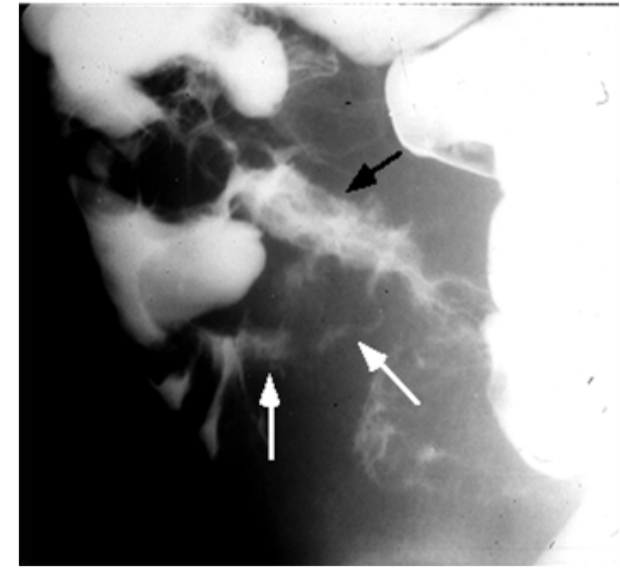

Figure 3b. Black arrow showing terminal ileum. White arrows showing ileocecal fistula.

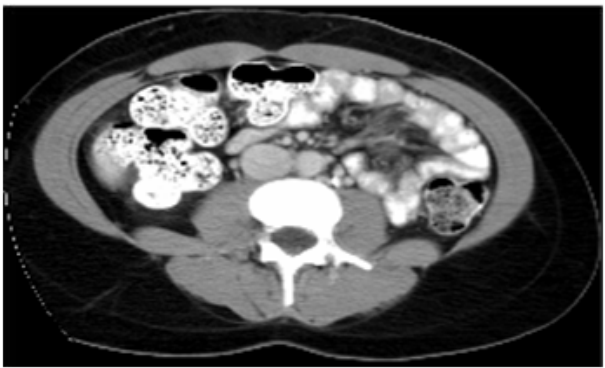

Figure 3c. Creeping fat

\section{Chris is experiencing?}

Answer: The distal ileum has specific receptors for the absorption of bile acids. However, when most of the terminal ileum is inflamed, as is the case with $\mathrm{CD}$, malfunctioning of the receptors ensues, and we get malabsorption of bile salt/acid that enter the colon. This results in stimulation of colonic enterocyte, leading to chloride secretion and water losses which in turn results in watery diarrhea. The case could be expanded to highlight complications that could be associated with the disease

\section{Why do you think Chris has iron deficiency anemia?}

Answer: Patients with $\mathrm{CD}$ can cause chronic blood loss from complications associated with $\mathrm{CD}$ e.g. ulcers, open sores and anal fissures. Also, due to the chronic inflammatory state, there will be suppression of erythropoietin production and alteration in iron metabolism, a condition called "anemia of chronic disease". In addition, involvement of the intestine in $\mathrm{CD}$ can lead to iron malabsorption, along with other vitamins and minerals.

11. Name at least three complications that Chris might face in the future.

Answer: Complications associated with $\mathrm{CD}$ include the following: strictures, fistulas, perianal disease, malabsorption, nutritional depletion, colorectal cancer, and calcium oxalate nephrolithiasis.

\section{Step 6.}

The sixth step involves discussion of the case by covering the requirements specified in the objectives. Additional resources like colonoscopy photos (normal vs abnormal), radiographs of the GI, and gastroscopy photos enrich the discussion. 


\section{Step 7.}

The session will terminate with an evaluation by the students of:

1. the process

2. the facilitators

3. the content, leaving some space for comments.

\section{Discussion}

The IBD case is an excellent prototype to study the whole GIT histology and histopathology. In our experience, the students provided an excellent overall evaluation. The great majority praised the strategies used and the close and beneficial integration between histology and histopathology. They reported that the case emphasized and enhanced self-learning and problem solving. The process stimulated development of interpersonal skills in approaching with a clinical scenario, very much like real situations. In addition, the case enhanced critical thinking and fostered team work, the basis for any successful physician.

There are many challenges to the study of medicine these days: Too much information, too little time, too many students, and reduction in student-faculty contact hours. In this context, resources can become the primary teacher while encouraging self-learning, and designing the appropriate strategies and approaches.

Both histology and histopathology have a major practical component to study slides. Both courses constitute a fundamental part of all biomedical curricula: They bridge the macroscopic field of gross anatomy and pathology to molecular sciences, to biochemistry and pharmacology among others. They actually constitute a central link between visible and submicroscopic dimensions. They do constitute the geography where normal and abnormal clinical manifestations take place. Using IBD constitutes an appropriate choice to study the GI tract since that study can involve any segment of the digestive system. In addition, IBD is associated with extra-intestinal manifestations, including the hepatobiliary system, the kidneys, the genitourinary tract, the endocrine system, the musculoskeletal system, the eyes and the skin. Thus, IBD constitutes a very good candidate for integrated teaching because of the extensive involvement of various disciplines from basic science to clinical disciplines such as anatomy, histology, physiology, histopathology, immunology, microbiology, biochemistry, pharmacology and others.

Medical curricula are likely to continue to become more integrated and clinically-based in the future. Cases like IBD should be developed more and more to stress self-learning, problem solving, and interpersonal skills in approaching and dealing with real situations. Such cases will lead to more collaboration on the part of faculty members from different disciplines.

\section{References}

1. General Medical Council (2002) Tomorrow's doctors: recommendations on undergraduate medical education. London: GMC.

2. Walsh K (2013) Oxford Textbook Of Medical Education. 1st ed. New York: Oxford University Press; 2013: 281.

3. Hassan S (2013) Concepts of vertical and horizontal integration as an approach to integrated curriculum. Education in Med J: 5(4).

Copyright: (C2017 Hussein IH. This is an open-access article distributed under the terms of the Creative Commons Attribution License, which permits unrestricted use, distribution, and reproduction in any medium, provided the original author and source are credited. 